\title{
PRESENCIA DEL OSO ANDINO TREMARCTOS ORNATUS (CARNIVORA: URSIDAE) EN EL BOSQUE TROPICAL AMAZÓNICO DEL PERÚ
}

\author{
JUDITH FIGUEROA ${ }^{1,2}$ \\ ${ }^{1}$ Asociación para la Investigación y Conservación de la Biodiversidad (AICB). \\ Av. Vicús 538. Lima 33. Perú. \\ ${ }^{2}$ Grupo de Investigación de Zoología de Vertebrados. Universidad de Alicante. España. \\ $<$ aicb.peru@gmail.com>.
}

Figueroa, J. 2012. Presencia del oso andino Tremarctos ornatus (Carnivora: Ursidae) en el bosque tropical amazónico del Perú. Acta Zoológica Mexicana (n. s.), 28(3): 594-606.

RESUMEN. En el Perú, el oso andino (Tremarctos ornatus) habita principalmente en el bosque montano, entre los 1900 y 2350 msnm. En contraposición, los registros del oso en el bosque tropical amazónico (BTA), debajo de los $650 \mathrm{msnm}$, son considerados como raros. Se evaluó la presencia de esta especie en el BTA en el límite del Parque Nacional Yanachaga Chemillén con la Reserva Comunal Yanesha (PNYC-RCY, $32.8 \mathrm{~km}$ ), en febrero/2005 y julio/2007, y en la Reserva Comunal Amarakaeri (RCA, $190.96 \mathrm{~km}$ ) en abril-mayo y agosto/2008. Para el primer caso, se encontró una abundancia relativa del oso andino, de 0.081 registros $/ \mathrm{km}$ en la época de lluvias y de 0.829 registros $/ \mathrm{km}$ en la época de estiaje. En el segundo, fue ausente en la época de lluvias y 0.032 registros $/ \mathrm{km}$ en la época de estiaje. Su presencia en el BTA durante la época de estiaje, estaría relacionada al consumo de las palmeras (Arecaceae), ya que la maduración de los frutos de los cuales se alimenta en los bosques premontano y montano se realiza en la época de lluvias. Además, en el PNYC-RCY, el BTA sería utilizado como un corredor para los desplazamientos del oso hacia otras áreas montañosas, aprovechando el menor caudal de los ríos debido a la disminución de las lluvias.

Palabras clave: oso andino, oso de anteojos, bosque tropical amazónico, distribución, Perú.

Figueroa, J. 2012. Andean bear Tremarctos ornatus (Carnivora: Ursidae) presence in the Amazon tropical forest of Peru. Acta Zoológica Mexicana (n. s.), 28(3): 594-606.

ABSTRACT. In Peru, the Andean bear (Tremarctos ornatus) inhabit primarily montain forest, between 1900 to $2350 \mathrm{~m}$ of altitude. In contrast, the records in the Amazon tropical forest (BTA), under $650 \mathrm{~m}$ of elevation, are rare. The presence of this species was evaluated in the BTA of the boundaries of the Yanachaga Chemillén National Park with the Yanesha Communal Reserve (PNYC-RCY, $32.8 \mathrm{~km}$ ), during the months of February/2005 and July/2007, and the Amarakaeri Communal Reserve (RCA, $190.96 \mathrm{~km}$ ) during the months of April-May and August/2008. In the first case, a relative abundance of $0.081 \mathrm{records} / \mathrm{km}$ during the rainy season and $0.829 \mathrm{records} / \mathrm{km}$ during the dry season. In the second, the Andean bear was absent during the rainy season and of 0.032 records $/ \mathrm{km}$ in the dry season. The presence

Recibido: 11/07/2012; aceptado: 26/09/2012. 
in the BTA during the dry season, could be related to the consumption of palms (Arecaceae), because the fruits that are the base of its diet in the montane and premontane forest are mature in the rainy season. In addition, at PNYC-RCY, the BTA could be used as a corridor for the movement of the Andean bear to other montane areas, taking advantage of the low water level on the river due to the lack of rain.

Key words: Andean bear, spectacled bear, amazon tropical forest, distribution, Peru.

\section{INTRODUCCIÓN}

El oso andino u oso de anteojos (Tremarctos ornatus), es la única especie de la familia Ursidae que habita en América del Sur, a lo largo de la Cordillera de los Andes, con poblaciones residentes desde Venezuela hasta Bolivia (Peyton 1999) y con algunos registros en Panamá (Elmore 1939, Jorgenson 1984, Goldstein et al. 2008) y el norte de Argentina (Del Moral \& Bracho 2005). En el Perú se distribuye entre los 210 y $4750 \mathrm{msnm}$, abarcando un área aproximada de $82,200 \mathrm{~km}^{2}$ (Patton et al. 1982, Peyton 1999), en donde ocupa diversos ecosistemas, como el bosque seco ecuatorial $(<1500$ $\mathrm{msnm})$, el bosque tropical amazónico $(<700-800 \mathrm{msnm})$, el bosque seco interandino $(<2000 \mathrm{msnm})$, el bosque tropical del Pacífico $(<1000 \mathrm{msnm})$, el bosque premontano (800-1800 msnm), el bosque montano (1800-3400 msnm), la puna (3400-5000 ms$\mathrm{nm})$ y el páramo (>3500 $\mathrm{msnm})$. En estos ecosistemas aprovecha todos los recursos disponibles, alimentándose de una gran variedad de especies botánicas (Figueroa \& Stucchi 2009).

De estos hábitats el oso andino muestra preferencia por el bosque montano, entre los 1900 y 2350 msnm, debido a la mayor disponibilidad de los frutos de los cuales se alimenta; así como por el bosque seco ecuatorial, en la cordillera occidental de los Andes, a partir de los 250 msnm, cuando hay disponibilidad de agua (Peyton 1980). En contraposición, sus registros en el bosque tropical amazónico, en la cordillera oriental de los Andes, son considerados como raros (Peyton 1999) (Cuadro 1).

El bosque tropical amazónico, también denominado bosque húmedo tropical, selva baja o llanura amazónica (Onern 1976, Brack 1986), se caracteriza por ser uno de los ecosistemas de mayor complejidad estructural, estratificación y diversidad de especies en el mundo (Etter 1998). Presenta un clima extremadamente húmedo y caluroso, que si bien no constituye el hábitat "típico" del oso andino, no debería ser excluyente para su vida, debido a la evidencia histórica y geográfica (Yerena 1987). $\mathrm{Al}$ respecto, algunos autores asumen que esta especie apareció en América Central de un antepasado que provino de América del Norte (Kurtén 1966), ingresando a América del Sur por Panamá hace 15,000 a 25,000 años atrás (Ruiz-García 2003). En este país, el oso andino ha sido registrado desde aproximadamente los $200 \mathrm{msnm}$ (López de Gómara 1922 [1510-1560], Elmore 1939).

Por ello, es posible que los pocos registros de la especie en el bosque tropical amazónico sean consecuencia de los escasos estudios que se han desarrollado sobre el mismo en este tipo de ecosistema. En la mayoría de los casos, se trata de reportes ocasionales de inventarios generales de mastofauna (Emmons \& Pacheco 1997, Borman 
Figueroa: El oso andino en el bosque tropical amazónico

Cuadro 1. Registros del oso andino en el bosque tropical amazónico y orinocense.

\begin{tabular}{clcl}
\hline País & \multicolumn{1}{c}{ Área } & $\begin{array}{c}\text { Altitud } \\
\text { msnm }\end{array}$ & \multicolumn{1}{c}{ Fuente } \\
\hline Panamá & Entre Nombre de Dios y Panamá & 200 & López de Gómara 1922 [1510-1560] \\
& Serranía de Darién & $200-300$ & Elmore 1939 \\
Venezuela & Río Orinoco & 200 & Vespucio 1992 [1503?] \\
& Caño Pimichín, orillas del río Temi & 100 & Humboldt 1985 [1800] en Yerena 1987 \\
& Selvas de la Guayana & 251 & Codazzi 1960 [1818-1848] en Yerena \\
& & & 1987 \\
Colombia & Rarare, Estado Apure & 400 & Yerena 1987 \\
& Ríos Uribante y Caparo & 300 & Goldstein 2006 en Goldstein et al. 2008 \\
Ecuador & Serranía de las Quinchas & 300 & Jorgenson 1980 en Yerena 1987 \\
& Serranía Cofán, Bermejo & 500 & Ojeda \& Pesca 2006 \\
& Cordillera del Kutukú & 450 & Borman 2002 \\
& Río Cofanes, Sucumbios & 500 & Zapata-Ríos et al. 2006 \\
& Entre los ríos Cenepa y Huampami & 210 & Patton et al. 1982, Patton 1999 \\
& (Amazonas) & & \\
& Puesto de Vigilancia Comainas, & 650 & Emmons \& Pacheco 1997 \\
& Cordillera del Cóndor (Amazonas) & & \\
& Parque Nacional Cordillera Azul & 600 & Rojas-Vera Pinto 2011 \\
& (San Martín) & & \\
& Región del bajo Urubamba (Cusco) & $400-700$ & Boddicker et al. 2009 \\
& Sandia (Puno) & 650 & Peyton 1980 \\
& Parque Nacional Bahuaja Sonene & 750 & Ascorra 2002, comunicación personal, \\
(entre Puno y Madre de Dios) & & en Figueroa \& Stucchi 2009 \\
& Parque Nacional Amboró & 550 & Rumiz et al. 1999 \\
\hline \multirow{5}{*}{ Bolivia } & &
\end{tabular}

2002, Zapata-Ríos et al. 2006, Borman \& Quenamá 2009, Boddicker et al. 2009). El objetivo de este trabajo fue registrar la presencia del oso andino en el bosque tropical amazónico en el centro y sur del Perú, y discutir las razones por las cuales se encontraría en este tipo de ambiente.

\section{MATERIALES Y MÉTODOS}

Área de estudio. La primera evaluación se llevó a cabo en el Parque Nacional Yanachaga Chemillén (PNYC), que se localiza en la región Pasco (selva central del Perú). Está ubicada entre los 340 y $3800 \mathrm{msnm}$ y tiene una extensión de $1220 \mathrm{~km}^{2}$, donde aproximadamente el 10\% está conformado por el bosque tropical amazónico (BTA) 
(Inrena 2006). Para el presente estudio, se recorrió el BTA del sector Paujil (10¹9’S y $75^{\circ} 15^{\prime} \mathrm{W}$ ), que limita con la Reserva Comunal Yanesha (RCY), entre los 340 y $700 \mathrm{msnm}$ (Fig. 1). Esta área se caracteriza por tener una temperatura y humedad promedio anual, de $25^{\circ} \mathrm{C}$ y $75 \%$, respectivamente, con precipitaciones anuales que varían entre 2000 y $6000 \mathrm{~mm}$ (Inrena 2006). Los bosques del sector Paujil, son por lo general siempre verdes, con un dosel forestal de $30 \mathrm{~m}$ y árboles emergentes que llegan hasta los $45 \mathrm{~m}$ de altura. Entre las familias registradas más importantes, tanto de hábito arbóreo y arbustivo como herbáceo, están Anacardiaceae, Annonaceae, Apocynaceae, Araceae, Araliaceae, Arecaceae, Bignoniaceae, Burseraceae, Cyatheaceae, Euphorbiaceae, Fabaceae, Heliconiaceae, Lauraceae, Malvaceae, Melastomataceae, Moraceae, Myristicaceae, Piperaceae, Rubiaceae, Sapotaceae, Siparunaceae, Urticaceae y Vochysiaceae. Entre las palmeras destacan Iriartea deltoidea, Astrocarium huicungo, Geonoma spp., Catoblastus sp., Euterpe precatoria, Oenocarpus bataua y Phytelephas macrocarpa (Vásquez et al. 2005).

La segunda evaluación se realizó en la Reserva Comunal Amarakaeri (RCA) ( $12^{\circ} 47^{\prime} \mathrm{S}$ y $\left.70^{\circ} 57^{\prime} \mathrm{W}\right)$ que pertenece a la región Madre de Dios (sureste del Perú). Está ubicada entre los 300 y $2700 \mathrm{msnm}$ y tiene una extensión de $4,023.35 \mathrm{~km}^{2}$, donde el 50\% está conformado por el BTA (Inrena 2009). Para el presente estudio, se recorrieron áreas ubicadas entre los 320 y $700 \mathrm{msnm}$ (Fig. 2), donde abundan

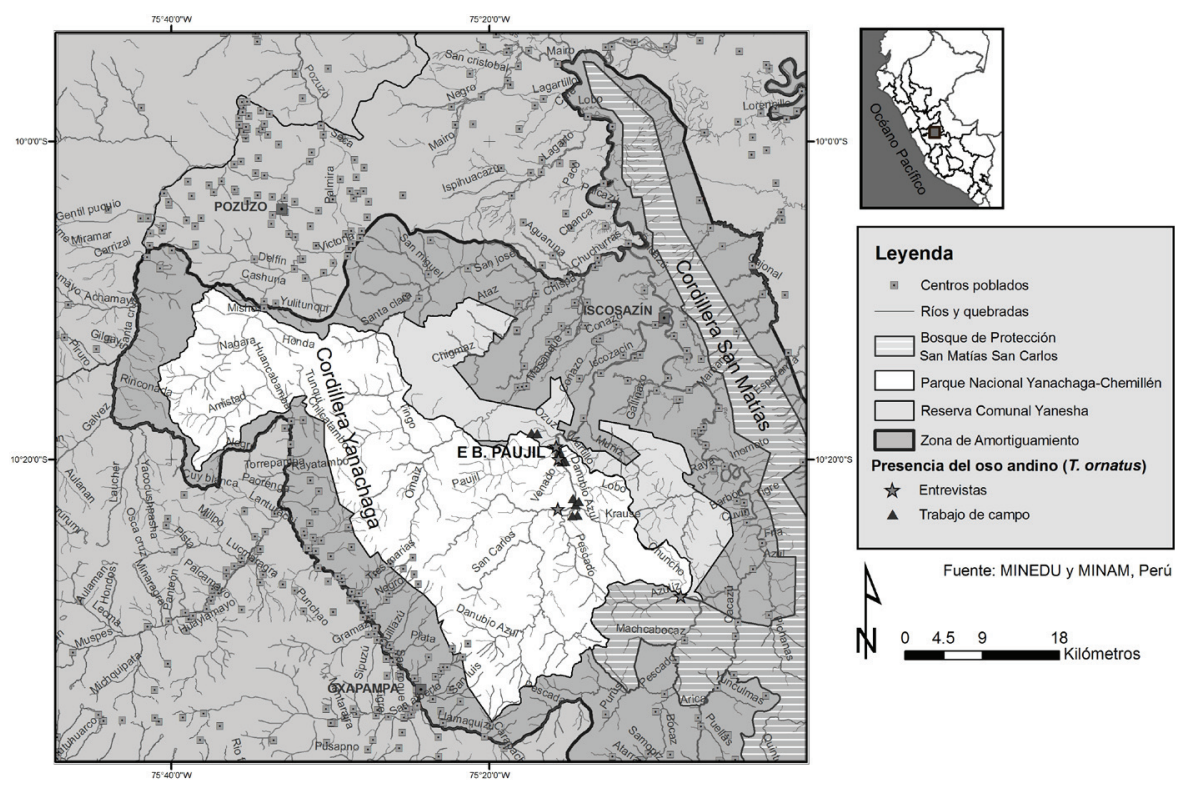

Figura 1. Registros del oso andino en el bosque tropical amazónico del Parque Nacional Yanachaga Chemillén y Reserva Comunal Yanesha. 


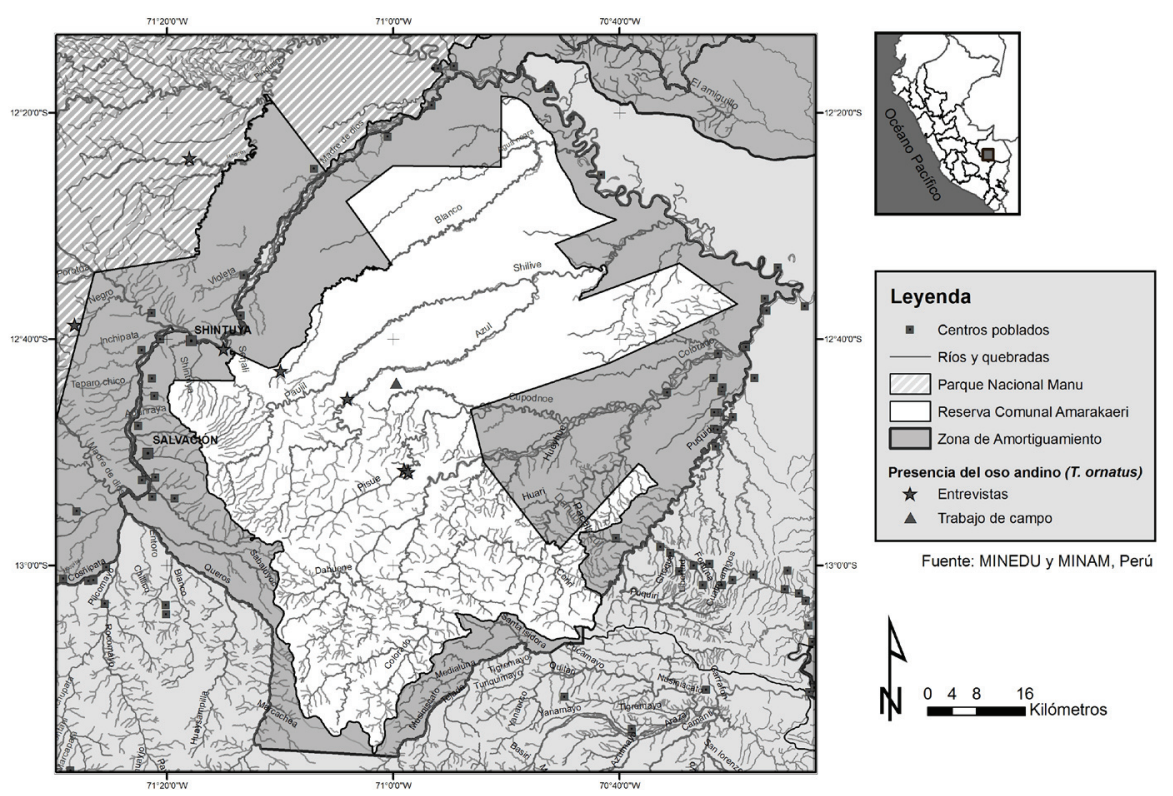

Figura 2. Registros del oso andino en el bosque tropical amazónico de la Reserva Comunal Amarakaeri.

especies vegetales de las familias Acanthaceae, Annonaceae, Araceae, Arecaceae, Euphorbiaceae, Fabaceae, Malvaceae, Melastomataceae, Meliaceae y Rubiaceae. Entre las palmeras destacan Astrocaryum murumuru, Euterpe precatoria, Geonoma macrostachys, Iriartea deltoidea y Socratea exorrhiza. Esta área se caracteriza por presentar una temperatura entre $\operatorname{los} 25^{\circ} \mathrm{C}$ y $38^{\circ} \mathrm{C}$, con una humedad y precipitación promedio anual de $90.3 \%$ y $3810 \mathrm{~mm}$, respectivamente (Inrena 2009). En las áreas evaluadas, las mayores precipitaciones (época de lluvias) se realizan entre diciembre a marzo, y las menores (época de estiaje) entre abril y noviembre.

Métodos. En el PNYC-RCY se evaluaron $12.3 \mathrm{~km}$ entre los días 15 al $25 \mathrm{de}$ febrero de 2005 (época de lluvias) y $20.5 \mathrm{~km}$ entre el 12 y 30 de julio de 2007 (época de estiaje). En la RCA se recorrieron $65.69 \mathrm{~km}$ entre el 13 de abril y 17 de mayo de 2008 (época de lluvias) y $125.27 \mathrm{~km}$ entre el 28 de julio y 22 de agosto de 2008 (época de estiaje). El trabajo de campo se realizó entre las 8:00 a.m. y 5:00 p.m. Se abrieron senderos de $1 \mathrm{~m}$ de ancho, los cuales se recorrieron a una velocidad aproximada de $1.5 \mathrm{~km} / \mathrm{h}$, observando cuidadosamente desde el dosel hasta el suelo para detectar la presencia de los posibles rastros dejados por el oso andino, como huellas, rasguños, restos de alimentación, caminos, madrigueras, pelos y excrementos; anotándose la posición geográfica y la altitud. También se registró la presencia de otros mamíferos grandes en las áreas donde se encontraron rastros del oso andino. La abundancia 
relativa (AR), se obtuvo dividiendo el número de registros del oso entre la distancia recorrida, de forma independiente para cada época evaluada (Figueroa 2004). Adicionalmente, se entrevistó a los guardaparques de las áreas protegidas visitadas, así como a los pobladores Yaneshas de Iscozasín (PNYC-RCY) y a los pobladores Harakmbut de las comunidades de Shintuya y Huasaroquito (RCA).

\section{RESULTADOS}

\section{Parque Nacional Yanachaga Chemillén-Reserva Comunal Yanesha}

Durante la época de lluvias, solo se encontró en el Mirador de Paujil (10¹9'57'’S y $75^{\circ} 15^{\prime} 37^{\prime}$ 'W $631 \mathrm{msnm}$ ) senderos de oso que no presentaron un uso reciente. Sin embargo, en la época de estiaje se encontraron 17 rastros frescos entre los 370 y 635 msnm, cerca del río Danubio Azul y las quebradas Krause y Pescado (dentro del PNYC), y la quebrada Osuz (dentro de la RCY). Estas consistieron en senderos, huellas, así como rasguños por marcaje de territorio en la corteza de árboles de Tachigali sp., Ormosia sp., Macrolobium gracile (Fabaceae) y Virola sp. (Myristicaceae), y rasguños por trepar árboles de M. gracile, Inga sp. (Fabaceae), Guatteria boliviana (Annonaceae), Meliosma sp. y Pouteria sp. (Sapotaceae). También se encontraron restos de alimentación de los frutos y médula de Wettinia longipetala y Geonoma sp. (Arecaceae). La AR del oso andino en la época de lluvia fue de 0.081 registros $/ \mathrm{km}$ y en la época de estiaje de 0.829 registros $/ \mathrm{km}$. Por otro lado, en las áreas donde se encontraron rastros del oso, también se registraron otras especies de mamíferos como el machín negro (Cebus apella), el mono choro común (Lagothrix lagotricha), el puerco espín (Coendou bicolor), el jaguar (Panthera onca), la nutria de río (Lontra longicaudis), la huangana (Tayassu pecari), el sajino (Pecari tajacu), el tapir amazónico (Tapirus terrestris) y el venado colorado (Mazama americana). Se observaron rastros del jaguar por toda el área evaluada, incluso al regreso de nuestros recorridos se encontraron sus huellas al lado de las nuestras, y se escucharon sus rugidos durante la noche en el campamento en la quebrada San Carlos. La AR del jaguar en la época de lluvias fue de 0.407 registros $/ \mathrm{km}$ y en la época de estiaje de 0.927 registros $/ \mathrm{km}$.

En las entrevistas, los guardaparques comentaron que el jaguar había atacado a un perro que acompañaba a dos niños Yaneshas que se dirigían a la comunidad de Osuz, una semana antes de nuestra evaluación. Asimismo, en el año 1988 los guardaparques trataron de domesticar a un oso joven que encontraron en el Mirador de Paujil, llevándole diariamente frutas, sin embargo, al intentar construirle un refugio, después de una semana, se marchó del área. También, en diciembre de 2001 observaron huellas y bromelias terrestres comidas por el oso cerca de la quebrada San Carlos. En Iscozasín, nos señalaron que en varias oportunidades, durante la época de estiaje, se han encontrado las médulas de ungurahui (Oenocarpus bataua, Arecaceae) comidas por 
el oso cerca de la quebrada Azulís, en el límite del PNYC con el Bosque de Protección San Matías San Carlos (10²8’34”S y 7507’56”W, 654 msnm) (Fig. 1).

\section{Reserva Comunal Amarakaeri}

Durante la época de lluvia no se observaron rastros del oso andino en el área de estudio. En la época de estiaje, se encontraron restos de alimentación frescos de Tillandsia sp., Guzmania sp. (Bromeliaceae epífita) y Asplundia sp. (Cyclanthaceae epífita), así como troncos doblados a lo largo de un sendero (1243'36”'S y 7059'41'”W, 385 $\mathrm{msnm}$ ). Estos presentaron una AR de 0.032 registros $/ \mathrm{km}$. Adyacente al área donde se encontraron los rastros, se registraron otras especies de mamíferos como el armadillo (Dasypus sp.), el oso bandera (Myrmecophaga tridactyla), el pichico común ( $\mathrm{Sa}$ guinus fuscicollis), C. apella, el majaz (Cuniculus paca), el puma (Puma concolor), $P$. onca, P. tajacu, T. terrestris y M. americana. Al igual que en el PNYC-RCY, se encontraron huellas del jaguar paralelas a las nuestras e incluso, se nos comentó que un mes antes de nuestra visita, este había atacado a un colono que fue a pescar a la zona. La AR del jaguar en la época de lluvias fue de 0.365 registros $/ \mathrm{km}$ y en la época de estiaje de 0.543 registros $/ \mathrm{km}$.

Según las entrevistas, cuando parte de la población Harakmbut vivió en las orillas del río Azul hasta mediados del siglo XX, observaron osos andinos cruzando este río, principalmente en la época de estiaje, siendo eventualmente cazados para el consumo de su carne. En 1978, un oso fue cazado en Shintuya cerca al río Madre de Dios. También se reportó la presencia reciente de machos y una hembra con cría entre los 390 y $470 \mathrm{msnm}$, entre marzo y octubre de 2007, en las zonas aledañas a los ríos Cupodnoe, Azul y Blanco, donde el oso se alimentó de la médula y frutos de ungurahui. En áreas aledañas a Shintuya, que se encuentran dentro del Parque Nacional Manu, también se ha registrado al oso andino. En el 2003, un oso le dio un zarpazo en el brazo a un ni-

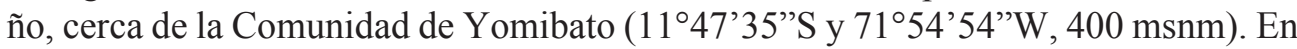
marzo de 2005, en la quebrada Gallinazo (12 $24^{\prime} 00^{\prime \prime S}$ y $71^{\circ} 17^{\prime} 58^{\prime \prime} \mathrm{W}, 408 \mathrm{msnm}$ ) se encontró restos de la médula de Bactris sp. e Iriartea deltoidea (Arecaceae) comidos por el oso. En abril de 2007, se observó un individuo caminando a las 6:00 p.m. cerca del límite con Palotoa (12³7’36’S y 71²8’03”W, 698 msnm) (Fig. 2).

\section{DISCUSIÓN}

\section{Uso del bosque tropical amazónico}

Peyton (1999) señaló que el oso andino no es residente permanente del BTA, siendo encontrado raramente por debajo de los $650 \mathrm{msnm}$. En base a los resultados aquí presentados, se piensa que, si bien esta especie ciertamente no es residente permanente, no se puede considerar rara su presencia en los BTA evaluados. En este trabajo se 
propone que el oso andino visita este hábitat de forma estacional, principalmente durante la época de estiaje. Esto concuerda con la época de sus registros en el bajo Urubamba (Perú) a 465 msnm, en el mes de mayo (Boddicker et al. 2009), y en Bermejo (Ecuador) a $450 \mathrm{msnm}$, entre julio y agosto (Borman 2002).

Teniendo en cuenta que el oso andino utiliza los hábitats de acuerdo con la oferta alimenticia (Peyton 1980), aprovechando todos los recursos que el bosque le provee cuando las condiciones climáticas le son favorables — que podría estar relacionado a una menor cantidad de lluvias, lo que significaría menos deslizamientos de tierras y crecidas intempestivas de los ríos, así como menores temperaturas-, en este trabajo se plantea que se desplazaría en la época de estiaje hacia el BTA en busca de otros recursos alimenticios, cuando la maduración de los frutos de los cuales se alimenta en los bosques premontano y montano disminuye. Estas fuentes de alimento en el BTA y en su límite con el bosque premontano, serían básicamente las palmeras (Arecaceae), cuyos frutos tienen altos niveles de energía, siendo ricos en carbohidratos, proteínas y grasas. Asimismo, la médula (cogollo), aunque es baja en calorías, tiene una digestión rápida (INS 2009), por lo que su consumo en altas cantidades cubriría las necesidades del oso. Esta familia ha sido registrada anteriormente como un componente importante en la dieta de esta especie en otras áreas a altitudes menores de 1000 msnm (Figueroa 2004, Ojeda \& Pesca 2006). Asimismo, en el BTA el oso andino podría tener un consumo ocasional del C. paca y Mazama sp., especies que han sido registradas como parte de su dieta a mayores altitudes (Herrera et al. 1994, Poveda 1986).

En la época de estiaje, debido a la disminución de las lluvias, los ríos bajan su caudal, no significando una barrera para el desplazamiento del oso andino a otras áreas en busca de alimento o traslado hacia otras montañas. Esto estaría acorde con lo señalado por los Harakmbut en la RCA sobre los avistamientos de esta especie cruzando los ríos, así como las huellas de individuos solitarios e incluso hembras con crías, encontradas en las playas.

Según Yerena (1987), en Venezuela, la población del oso andino se presenta básicamente en dos regiones que actualmente se encuentran separadas por llanuras de baja altitud con bosques húmedos, pantanos y sabanas. Esto sugiere que los individuos que se trasladaron al oriente tuvieron que haber cruzado estas zonas bajas, que en la actualidad están transformadas en áreas agrícolas, lo que constituye una barrera real que les impide regresar. En el caso de las áreas evaluadas en el PNYC-RCY y RCA, estas no están fuertemente impactadas por la intervención humana, a pesar de existir algunos centros poblados menores en su zona de amortiguamiento, por lo que se observan bosques continuos donde el oso andino puede trasladarse hacia otras áreas.

En base a la abundancia relativa del oso andino en la época de estiaje en el PNYC-RCY (0.829 registros $/ \mathrm{km})$ y la RCA (0.032 registros $/ \mathrm{km})$, este presenta un mayor uso del BTA en el primero. En el caso del PNYC-RCY, el BTA del sector Paujil se 
encuentra rodeado por bosques premontanos, de los cuales los registros más distantes de la especie estuvieron en un máximo de $8 \mathrm{~km}$, por lo que es posible que algunos individuos utilicen el área como un corredor entre la cordillera de Yanachaga y la cordillera San Matías (Bosque de Protección San Matías San Carlos), en donde, en ambos lados, se ha registrado a la especie (Figueroa \& Stucchi 2009). En su traslado se alimentarían principalmente de las palmeras y de otras fuentes que el bosque le provee. Por otro lado, en la RCA, el registro más distante del oso andino al bosque premontano se ubicó a $25 \mathrm{~km}$ hacia el este, continuando en esta misma dirección extensas áreas de BTA. Por ello, es probable que en la época de estiaje, algunos individuos visiten de forma eventual estas zonas en busca de alimento y exploración del territorio, como una continuación del bosque premontano.

\section{¿El jaguar limita la presencia del oso andino en el bosque tropical amazónico?}

Anteriormente se señaló que el oso andino parecía evitar al jaguar, ya que sus rangos altitudinales en el Perú y Bolivia no se superponían en la ladera de una misma montaña (Peyton 1999). Sin embargo, en este estudio se determinó que en las mismas áreas donde se registró al oso andino en el PNYC-RCY y la RCA, se encontró una alta actividad del jaguar, presentando una mayor abundancia relativa que el oso. Incluso en el PNYC, en un árbol de Ormosia sp. (Fabaceae) se encontró marcajes de territorio tanto del oso andino como de un felino, que muy posiblemente correspondería a un jaguar.

Esto nos sugiere que la presencia del jaguar no influiría en el desplazamiento del oso andino hacia y en el BTA, al menos en las zonas evaluadas y de forma directa. Aunque se ha documentado que el puma y el jaguar se evitan mutuamente en la región del pantanal y de los llanos, usando este último áreas con cobertura densas más que los pumas (Schaller \& Crawshaw 1980, Emmons 1987, Scognamillo et al. 2003), esto no ocurriría con el oso andino, debido a que este se alimenta en las zonas de traslape con el jaguar (BTA y bosque premontano) básicamente de palmeras (Arecaceae) y otros frutos de Sapotaceae y Fabaceae, no siendo un competidor por alimento.

Este tipo de coexistencia entre el jaguar y el oso andino queda demostrada en el bosque premontano bajo, donde el primero se registra hasta los $1500 \mathrm{msnm}$ (Peyton 1999) y donde el oso andino presenta una explotación más frecuente que en el BTA, debido a que esta es un área de transición donde se empiezan a encontrar frutos de las familias Chloranthaceae, Clusiaceae, Ericaceae y Myrtaceae, abundantes en el bosque montano, de los cuales se alimenta. Si bien, en la RCA el oso andino no se había registrado anteriormente en el BTA, su presencia ya había sido reportada en altitudes bajas dentro del bosque premontano, entre los 800 y $1000 \mathrm{msnm}$ en el mes de septiembre, cuando aún no se inician las lluvias fuertes (Fernández \& Kirkby 2001), y de igual manera en el PNYC (datos no publicados). Por otro lado, también existen registros del oso en el bosque premontano dentro del Santuario Nacional Megantoni 
(Cusco) a 960 msnm (Figueroa 2004) y en la Cordillera de Colán (Amazonas) desde los 1000 msnm (Butchart et al. 1995).

Otro ejemplo de coexistencia entre un úrsido y el jaguar, existe en el bosque mesófilo de montaña (800-1400 msnm) en la Reserva de Biósfera El Cielo, en México, donde el oso negro (Ursus americanus) y el jaguar conviven en este hábitat marginal. Al parecer, ambas especies no presentarían competencia en esta área, ya que el jaguar se alimenta principalmente del ganado y el oso negro de los cultivos de maíz (Vargas \& Hernández 2001).

\section{Impacto humano}

Las crónicas de los siglos XVI al XVIII parecen indicar que la distribución del oso andino incluía las áreas del BTA en mayor grado que en la actualidad (López de Gómara 1922 [1510-1560], Yerena 1987, Vespucio 1992 [1503?]). Este cambio se podría haber originado debido a la transformación de estos hábitats por el hombre, por lo que algunas zonas bajas habrían dejado de proveer espacio y alimento al oso andino, así como de ser corredores naturales entre las áreas montañosas. Uno de los grupos más importantes en la dieta de la especie en el BTA y bosque premontano son las palmeras, las cuales han sido las más utilizadas por los humanos y por tanto, las más susceptibles de agotarse (Johnson 1998). Con el crecimiento de la población humana, este uso se ha ido intensificando, reduciendo la cantidad de palmeras disponibles para el oso andino. Por ejemplo, en la Serranía de las Quinchas (Colombia), en altitudes menores a los $1000 \mathrm{msnm}$, el oso andino compite con el hombre por los frutos de Oenocarpus mapora, una de sus principales fuentes de alimento en el área, debido a que es consumida por la gente como un reemplazo de la leche por su alto valor en proteína y aceite (Ojeda \& Pesca 2006).

En el caso del Perú, muchas áreas de bosques amazónicos se mantuvieron en buenas condiciones, entre otros factores, debido a la falta de vías de comunicación y a la guerra interna (terrorismo), que mantuvo a distancia a los colonos y extractores por décadas. No obstante, en la actualidad dentro de la RCA, existen otros peligros potenciales que podrían conllevar a diversos impactos negativos sobre la fauna y flora. Estos son la extracción maderera, la presencia de concesiones petroleras y el asentamiento de pequeños campamentos de extractores de oro en el sureste de la reserva, que podrían expandirse a otras zonas. Todas estas actividades generan ruidos fuertes, contaminación de las aguas, deforestación de los boques y cacería de animales silvestres para consumo. En el caso del PNYC-RCY, el área de BTA se encuentra bien conservada debido a su difícil acceso y a la ubicación estratégica de la Estación Biológica de Paujil, que les permite una vigilancia continua. Sin embargo, el establecimiento de innumerables, aunque aún pequeños, centros poblados en los alrededores de las áreas protegidas evaluadas (Figs. 1 y 2), en un futuro podrían formar una barrera, originando el aislamiento de algunas poblaciones de la fauna. 
Agradecimientos. A Avelino Sebastián, Tomás Ciriaco, Manuel Soto, Abad Utani, Samuel Enempa, Samuel Mankebe y Trinidad Tapia por su valioso apoyo en el trabajo de campo. A Abel Monteagudo por su ayuda en la identificación de las especies botánicas que formaron parte de la dieta del oso. A Marcelo Stucchi y Paul Velazco por sus valiosos comentarios al manuscrito. A Rocío Rojas y Rodolfo Vásquez del Centro para la Conservación y Desarrollo Sostenible del Jardín Botánico de Missouri, por el financiamiento y apoyo logístico para el trabajo de campo en la selva central. A Idea Wild por la donación de los equipos. A Gilmer Pretell y Humberto Cristóbal por compartir sus observaciones sobre la presencia del oso en las inmediaciones de Paujil. A Roxana Rojas por el diseño de los mapas. Al revisor anónimo por sus acertados comentarios.

\section{LITERATURA CITADA}

Boddicker, M. L., Rodríguez, J. J. \& Amanzo, J. 1999. Medium and Large Mammals: Biodiversity Assessment at the Pagoreni Well Site. Pp. 151-192. In: Alonso, A. and F. Dallmeier (Eds.). Biodiversity Assessment of the Lower Urubamba Region, Peru: Pagoreni Well Site Assessment and Training. SI/MAB Series 3. Smithsonian Institution/MAB Biodiversity Program. Washington, EEUU.

Borman, R. 2002. Mamíferos grandes. Pp. 76-81, 210-213. In: Pitman, N., D. K. Moskovits, W. S. Alverson, and R. Borman (Eds.). Ecuador: Serranías Cofán-Bermejo, Sinangoe. Rapid Biological Inventories, Report 3. The Field Museum. Chicago, EEUU.

Borman, R. \& Quenamá, A. 2009. Mamíferos. Pp. 99-106. In: Vriesendorp, C., W. S. Alverson, A. del Campo, D. F. Stotz, D. K. Moskovits, S. Fuentes, B. Coronel and E. P. Anderson (Eds.). Ecuador: Cabeceras Cofanes-Chingual. Rapid Biological and Social Inventories, Report 21. The Field Museum. Chicago, EEUU.

Brack, A. 1986. Ecología de un país complejo. Pp. 175-319. In: Manfer-Mejía Baca (Ed.). Gran Geografía del Perú. Madrid, España.

Butchart, S., Barnes, R., Davies, C., Fernández, M. \& Seddon, N. 1995. Threatened Mammals of the Cordillera de Colán, Peru. Oryx, 29: 275-281.

Del Moral, J. F. \& Bracho, A. E. 2005. Evidence of Andean bear in northwest Argentina. International Bear News, 14: 30-32.

Elmore, F. 1939. Colecta del cráneo de una hembra de oso andino en Panamá, el 4 de abril de 1939. Depositado en el Natural History Museum of Los Angeles County (catálogo 20107), EEUU.

Emmons, L. H. 1987. Comparative feeding ecology of felids in a neotropical rainforest. Behavioral Ecology and Sociobiology, 20: 271-283.

Emmons, L. H. \& Pacheco, V. 1997. Mamíferos del alto río Comainas. Pp. 78-82. In: Schulenberg, T. S. and K. Awbrey (Eds.). The Cordillera del Condor region of Ecuador and Peru: a biological assessment. Rapid Assessment Program - RAP Working Papers 7. Conservation International. Washington, EEUU.

Etter, A. 1998. Bosque húmedo tropical. Pp. 106-133. In: Chaves, M.E. and N, Arango. (Eds.). Informe nacional sobre el estado de la diversidad. Instituto de Investigación de Recursos Biológicos Alexander von Humboldt, PNUMA, Ministerio del Medio Ambiente. Bogotá, Colombia.

Fernández, M. \& Kirkby, C. 2002. Evaluación del estado poblacional de la fauna silvestre y el potencial turístico en los bosques de Salvación y Yunguyo, Reserva de Biósfera del Manu, Madre de Dios, Perú. Reporte Final. Proyecto Aprovechamiento y Manejo Sostenible de la Reserva de Biósfera y Parque Nacional del Manu - ProManu. Cusco, Perú. 59 pp.

Figueroa, J. 2004. Mamíferos. Pp. 110-118, 215-230, 288-293. In: Vriensedorp, C., L. Rivera, D. Moskovits and J. Shopland (Eds.). Perú: Megantoni. Rapid Biological Inventories, Report 15. The Field Museum. Chicago, EEUU. 
Figueroa, J. \& Stucchi, M. 2009. El Oso Andino: alcances sobre su historia natural. Asociación para la Investigación y Conservación de la Biodiversidad (AICB). Lima, Perú. 105 pp.

Goldstein, I., Guerrero, V. \& Moreno, R. 2008. Are there Andean bears in Panama? Ursus, 19: 185189

Herrera, A. M., Nassar, J., Michelangeli, F., Rodrígues, J. P. \& Torres, D. 1994. The spectacled bear in the Sierra Nevada National Park of Venezuela. International Conference on Bear Research and Management, 9: 149-156.

Inrena (Instituto Nacional de Recursos Naturales). 2006. Parque Nacional Yanachaga Chemillén: Plan Maestro 2005-2009. Lima, Perú. 220 pp.

Inrena. 2009. Reserva Comunal Amarakaeri: Plan Maestro 2008-2012. Lima, Perú. 215 pp.

INS (Instituto Nacional de Salud). 2009. Tablas peruanas de composición de alimentos. Ministerio de Salud. Lima, Perú. 64 pp.

Johnson, D. V. 1998. Non-wood forest products: tropical palms. FAO RAP Publication 10. 165 pp.

Jorgenson, J. 1984. Informe de los países: Colombia. Boletín del Grupo de Especialista del Oso Frontino, $7: 13$.

Kurtén, B. 1966. Pleistocene Bears of North America: Genus Tremarctos, spectacled bears. Acta Zoologica Fennica, 115: 1-96.

López de Gómara, F. 1922 [1510-1560]. Historia general de las indias. Tomo I. Editorial Calpe. Madrid, España. 255 pp.

Ojeda, M. C. \& Pesca, A. L. 2006. Uso del hábitat natural del oso andino (Tremarctos ornatus) en la Serranía de las Quinchas, Magdalena Medio (Colombia). Universidad Pedagógica y Tecnológica de Colombia. Tesis de Licenciatura 76 pp. (Inédita).

Onern (Oficina Nacional de Evaluación de Recursos Naturales). 1976. Mapa Ecológico del Perú: guía explicativa y mapas. Lima, Perú. 146 pp.

Patton, J. L. 1999. Mamíferos de la cuenca del río Cenepa. Pp. 77-78. In: Conservación Internacional (Ed.). Biodiversidad de la Cordillera del Cóndor: referencias técnicas para su conservación. Lima, Perú.

Patton, J. L., Berlin, B. \& Berlin, E. A. 1982. Aboriginal perspectives of a mammal community in amazonian Peru: knowledge and utilization patterns among the Aguaruna Jivaro. Pp. 111-128. In: M. A. Mares and H. H. Genoways (Eds.). Mammalian Biology in South America. Pymaturing Symposia in Ecology 6. Special Publication Series, Pymaturing Laboratory of Ecology, University of Pittsburgh. Pensilvania, EEUU.

Peyton, B. 1980. Ecology, distribution and food habits of spectabled bear, Tremarctos ornatus, in Peru. Journal of Mammalogy, 61: 639-652.

Peyton, B. 1999. Spectacled bear conservation action plan. Pp. 157-198. In: Servheen, C., S. Herrero and B. Peyton (Eds.). Bears: Status survey and conservation action plan. UICN/SSC Bear Specialist Group. Gland, Suiza y Cambridge, Reino Unido.

Poveda, F. 1986. El oso andino, especie para conservar. Carta Ganadera, 23: 37-43.

Rojas-Vera Pinto, R. 2011. Revalorando la geografía animal. Estudio del isnachi (Tremarctos ornatus) en el distrito de Chazuta, San Martín - Parque Nacional Cordillera Azul. Universidad Católica del Perú. Tesis de Licenciatura 135 pp. (Inédita).

Ruiz-García, M. 2003. Molecular population genetic analysis of the spectacled bear (Tremarctos ornatus) in the northern andean area. Hereditas, 138:81-93.

Schaller, G. B. \& Crawshaw, P. G. 1980. Movement patterns of jaguar. Biotropica, 12: 161-168.

Scognamillo, D., Matrix, I., Sunquist, M. \& Polisar, J. 2003. Coexistence of jaguar (Panthera onca) and puma (Puma concolor) in a mosaic landscape in the venezuelan llanos. Journal of Zoology, 259: $269-279$. 
Vargas, J. A. \& Hernández, A. 2001. Distribución altitudinal de la mastofauna en la Reserva de Biósfera "El Cielo", Tamaulipas, México. Acta Zoológica Mexicana, 82: 83 -109.

Vásquez, R., Rojas, R., Monteagudo, A., Meza, K., Van Der Werff, H., Ortiz-Gentry, R. \& Catchpole, D. 2005. Flora Vascular de la selva central del Perú: una aproximación de la composición florística de tres áreas naturales protegidas. Arnaldoa, 12: 112 - 125.

Vespucio, A. [1503?] 1992. El Nuevo Mundo: naturaleza y costumbres de aquella gente. Pp. 27-31. In: Becco, H. (Ed.). Historia real y fantástica del Nuevo Mundo. Fundación Biblioteca Ayacucho. Caracas, Venezuela.

Yerena, E. 1987. Distribución pasada y contemporánea de los úrsidos en América del Sur. Seminario EA - 7154. Universidad Simón Bolívar. Caracas, Venezuela. 32 pp.

Zapata-Ríos, G., Araguillin, E. \& Jorgenson, J. P. 2006. Caracterización de la comunidad de mamíferos no voladores en las estribaciones orientales de la Cordillera del Kutukú, Amazonia Ecuatoriana. Mastozoología Neotropical, 13: 227-238. 